\title{
Expression of the homophilic adhesion molecule, Ep-CAM, in the mammalian germ line
}

\author{
R. Anderson ${ }^{1,2}$, K. Schaible ${ }^{1}$, J. Heasman ${ }^{1,2}$, and C. Wylie ${ }^{1,3^{*}}$ \\ ${ }^{1}$ Institute of Human Genetics; ${ }^{2}$ Department of Cell Biology and Neuroanatomy; and ${ }^{3}$ Department of Pediatrics, \\ University of Minnesota, Minneapolis, MN 55455, USA
}

\begin{abstract}
During normal embryonic development, mammalian germ cells use both cell migration and aggregation to form the primitive sex cords. Germ cells must be able to interact with their environment and each other to accomplish this; however, the molecular basis of early germ cell adhesion is not well characterized. Differential adhesion is also thought to occur in the adult seminiferous tubules, since germ cells move from the periphery to the lumen as they differentiate. In a screen for additional adhesion molecules expressed by the germ line, expression of the homophilic adhesion molecule, Ep-CAM, was identified in embryonic, neonatal and adult germ cells using immunocytochemistry and flow cytometry with an Ep-CAM-specific monoclonal antibody. At embryonic stages, germ cells were found to express Ep-CAM during migration at embryonic day 10.5 and early gonad assembly at embryonic day 12.5 . Expression of Ep-CAM was also found on neonatal male and female germ cells. In the adult testis, Ep-CAM was detected only on spermatogonia, and was absent from more differentiated cells. Finally, embryonic stem cells were shown to express this receptor. It is proposed that Ep-CAM plays a role in the development of the germ line and the behaviour of totipotent cells.
\end{abstract}

\section{Introduction}

The germ line in mammals is established during the early stages of gastrulation. In mice, this corresponds to embryonic day 7.5. The precursors of the gametes are known as primordial germ cells (PGCs), and are morphologically similar in males and females. PGCs migrate from the hindgut endoderm to the mesoderm of the urogenital ridges to be incorporated into the future gonads (for review, see Wylie, 1999). After this migration, germ cells of both sexes aggregate with each other and with epithelial somatic cells. The clusters formed are the progenitors of the ovarian follicles in females or the seminiferous tubules in males, and are sexually dimorphic by embryonic day 12.5 (for review, see Anderson et al., 1998). In both adult males and females, germ cells are associated closely with a population of supporting somatic cells.

Cell-cell interactions are known to be required for germ cell survival in embryonic and adult mice (Nocka et al., 1989). Mouse PGCs will not migrate on plastic or purified extracellular matrix proteins, but will migrate on fibroblast feeder layers (Donovan et al., 1986). Although the cell-cell adhesion molecules necessary for germ cell development are not known, several of these types of receptor are known to be expressed in the germ line. Male germ cells have been shown to express $\mathrm{E}$ cadherin and $\mathrm{N}$ cadherin (Newton et al.,

*Correspondence

Received 19 November 1998.
1993; Wu et al., 1993). Expression of E cadherin is detected on mouse oocyte surfaces after fertilization or parthenogenetic activation (Clayton et al., 1995). mRNA encoding P cadherin has been detected in the testis of newborn rats (Lin and DePhilip, 1996), but is not required for fertility in mice (for review, see Hynes, 1996). N-CAM is not expressed in embryonic mouse gonads (Møller et al., 1991), but it has been reported to mediate gonocyte-Sertoli cell adhesion in neonatal rat testis (Orth and Jester, 1995). Neural cell adhesion molecule (N-CAM) is not required for fertility in mice (Cremer et al., 1994), so the role of this molecule in germ line development is not yet clear.

Recently, the pan-carcinoma marker known variously as EGP-40, KSA and ESA was shown to be a calciumindependent homophilic adhesion molecule, and was renamed Ep-CAM (Litvinov et al., 1994). Ep-CAM is not related structurally to other cell-cell adhesion molecules, although it does contain a nidogen-like domain as well as thyroglobulin- and epidermal growth factor-like repeats (Strand et al., 1989). Ep-CAM genes have been described in many vertebrate genomes (Linnenbach et al., 1993). The human and mouse forms of Ep-CAM are $86 \%$ identical at the amino acid level (Bergsagel et al., 1992). Ep-CAM expression was shown to be sufficient to induce clustering of $L$ cells and segregation of transfectants in mixed-cell aggregates in vitro (Litvinov et al., 1997). The role of Ep-CAM in vivo is not known, although experiments indicate that it plays a role in pancreatic islet morphoregulation and thymocyte function after stimulation (Nelson et al., 1996; Cirulli et al., 1998). 
In the present study, the expression of Ep-CAM by embryonic, neonatal and adult germ cells was investigated.

\section{Materials and Methods}

\section{Antibodies}

Purified G8.8, previously shown to bind murine Ep-CAM specifically (Nelson et al., 1996), was obtained from the Developmental Studies Hybridoma Bank, Iowa City, IA. Clone TG-1, a monoclonal mouse anti-SSEA-1 hybridoma, was a kind gift of P. Beverley (Division of Hematology, Department of Medicine, Cornell Medical School). TG-1 specifically labels germ cells in the early embryo (Donovan et al., 1986). Biotinylated anti-Forssman antigen (Fo) was purchased from Pharmingen, San Diego, CA. Fluorescein isothiocyanate (FITC)-anti-mouse IgM, FITC-anti-rat IgG, indocarbocyanine (Cy3)-anti-rat IgG, and phycoerythrin (PE)-streptavidin were purchased from Jackson Immunoresearch (West Grove, PA). The Forssman antigen is a specific marker for germ cells in the early gonad (Stinnakre et al., 1981). Polyclonal anti-laminin was purchased from Sigma (St Louis, MO), and used at a dilution of 1:500.

\section{Tissue culture}

STO cells (immortalized murine embryonic fibroblasts) were maintained as reported by Cooke (1993). The embryonic stem (ES) cell line, RA7 (50\% FVB, 50\% 129/J background; JAX mice) was derived and maintained in our laboratory using standard methods (Hogan et al., 1994). ES cells were plated on $\gamma$-irraditated clone D9 Ep-CAM+STO cells (see below).

\section{Sub-cloning and transfections}

The plasmid 5952, containing the full length Ep-CAM cDNA sequence, was a kind gift of L. Bergsagel (Department of Pathology, University College Hospital Medical School, London). The Ep-CAM coding sequence was removed from the vector by EcoRI digestion and subsequent gel purification, and was sub-cloned into the expression vector PCDNA3.1(-) (Invitrogen, Carlsbad, CA), which had been digested with EcoRI and treated with phosphatase. The resulting construct, pCDNA-EpCAM ${ }^{+}$, was linearized with BglII, extracted in phenol-choloroform and precipitated in ethanol. Next, $50 \mu \mathrm{g}$ of this construct was resuspended in $\mathrm{H}_{2} \mathrm{O}$ and transfected into STO fibroblasts by electroporation using a GenePulser II (BioRad). The electroporated cells were grown in $200 \mu \mathrm{g}$ G418 $\mathrm{ml}^{-1}$ (GIBCO, Gaithersberg, MD) for 2 weeks, and resistant colonies were picked, expanded and analysed selectively for stably transfected STO cells. Several G418-resistant STO cell colonies were found to express Ep-CAM, including clone D9, which was used in this study.

\section{Histology}

Mouse embryos were obtained from CD1 mice (Charles River, Wilmington, MA) and the morning of vaginal plug considered as day 0.5 of pregnancy. Sexing of embryos was performed by PCR (Hogan et al., B. 1994). For frozen sections, tissue was embedded in OCT (Tissue-Tek, Torrance, CA), snap-frozen in isopentane, submerged in liquid $\mathrm{N}_{2}$, and cut into $10 \mu \mathrm{m}$ sections. After brief fixation in acetone, slides were washed in PBS and blocked with PBS $+10 \%$ goat serum (PSA). G8.8 was diluted 1:1000 in PSA, and TG-1 was diluted 1:5 in PSA. After thorough washing in PBS and dilution in PSA (FIC 1:1000; Cy3 1:500), secondary antibodies were added. After several further washes in PBS, slides were mounted in $90 \%$ glycerol, $10 \% \mathrm{H}_{2} \mathrm{O}$ with $100 \mu \mathrm{g}$ 1,4-diazabicycl-o[2.2.2]octane (DABCO) ml ${ }^{-1}$ (Sigma). Sections were analysed with a Bio-Rad 1024 laser confocal microscope.

\section{Flow cytometry}

Cells were washed $\times 1$ with PBS, and subsequently incubated in PBS +10 mmol EDTA l-1 at $37^{\circ} \mathrm{C}$ for $15 \mathrm{~min}$ to assay parental and transfected STO fibroblasts for expression of Ep-CAM. Cells were then triturated into a single cell suspension, pelleted by centrifugation at $200 \mathrm{~g}$ for $5 \mathrm{~min}$ in a clinical centrifuge, and resuspended in $0.5 \mathrm{ml}$ PBS $+1 \%$ fetal calf serum (FCS; Sigma). The gonads of male and female embryonic day 12.5 embryos were dissected free of adjacent mesonephric tissue, disaggregated, pelleted and resuspended as above to analyse embryonic day 12.5 germ cells. Cells were incubated at $4^{\circ} \mathrm{C}$ with primary antibodies for $30 \mathrm{~min}$, pelleted as above and washed in $2 \mathrm{ml} \mathrm{PBS}+1 \%$ FCS, incubated with secondary antibodies for $30 \mathrm{~min}$, pelleted, and washed in $2 \mathrm{ml}$ PBS $+1 \%$ FCS for immunolabelling. After the final wash, cells were pelleted as above and resuspended in PBS $+1 \%$ FCS at a concentration of $2 \times 10^{6}$ cells $\mathrm{ml}^{-1}$. All antibodies used for flow cytometry were used at a dilution of $1: 100$ in PBS $+1 \%$ FCS. Flow cytometry was performed with a FacsVantage (Becton Dickinson, San Diego, CA) and analysed with Cellquest (Becton Dickinson).

\section{Results}

The aim of this study was to determine whether cells of the mammalian germ line express the homophilic adhesion molecule, Ep-CAM. For this purpose, G8.8, a monoclonal antibody shown to detect Ep-CAM on thymocytes and thymic epithelial cells (Nelson et al., 1996), was used. Studies with this antibody showed that STO fibroblasts were Ep$\mathrm{CAM}^{-}$by flow cytometry and immunfluorescent staining (Fig. 1; data not shown). However, upon stable transfection with a mouse Ep-CAM construct, several STO cell clones were found to be Ep-CAM ${ }^{+}$by flow cytometry and immunofluorescent staining (Fig. 1; data not shown). These experiments confirmed the use of G8.8 as a suitable marker for identification of Ep-CAM cells by flow cytometry and immunofluorescent staining in the system used.

Frozen sections of mouse embryos at stages of PGC migration (embryonic day 10.5) and germ cell coalescence (embryonic day 12.5) were double-stained with Ep-CAM- 


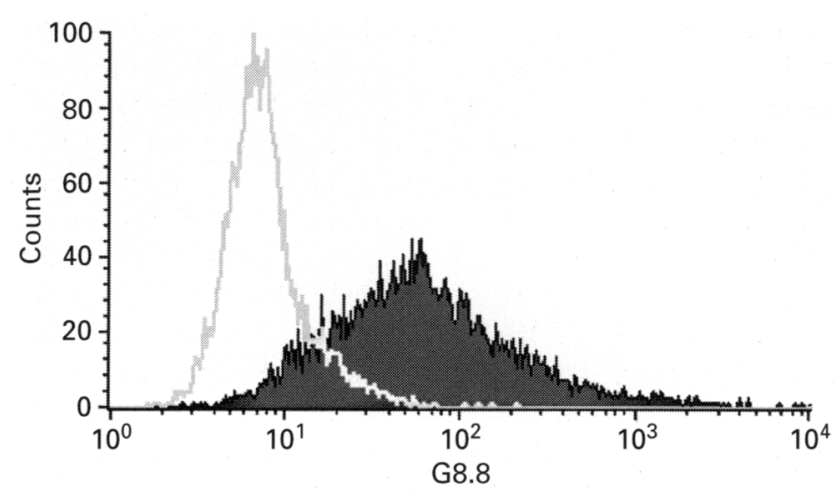

Fig. 1. Expression of the homophilic adhesion molecule, Ep-CAM, by transfected STO cell line D9. Flow cytometry scan of untransfected (grey curve) and clone D9 STO cells stably transfected with a mouse Ep-CAM expression construct (dark curve). G8.8 is a monoclonal antibody shown to detect Ep-CAM.

and germ cell-specific monoclonal antibodies to determine whether immunoreactive Ep-CAM is expressed in the early germ line. Expression of Ep-CAM was detected on the membranes of migratory PGCs (Fig. 2). In particular, anti-Ep-CAM heavily labelled dendrite-like processes of migratory PGCs (Fig. 2a,c). Ep-CAM+ PGC processes often extended $>15 \mu \mathrm{m}$ beyond the margins of the cell bodies (Fig. 2c). Ep-CAM expression was detected in the sex cords of embryonic day 12.5 male and female embryos (Fig. 2e-h), although expression was less prominent than at embryonic day 10.5. At embryonic day 12.5, mesonephric tubules were heavily labelled with the anti-Ep-CAM antibody (Fig. 2e,g).

Two-colour flow cytometry of embryonic day 12.5 male and female gonads was performed with germ cell- and Ep-CAM-specific monoclonal antibodies to confirm that membrane-bound Ep-CAM was on the surfaces of germ cells and not on neighbouring somatic cells. PGCs expressed more immunoreactive Ep-CAM than the majority of somatic cells of the early gonad (Fig. 2).

Immunoreactive Ep-CAM on germ cells was found in the gonads of male embryos and fetuses. At birth, germ cell expression of Ep-CAM was detected in the testis cords (Fig. 3a). In the adult testis, Ep-CAM was restricted to small round cells adjacent to the basement membrane of the seminiferous tubules, the spermatogonia (Fig. 3c,e). Few Ep$\mathrm{CAM}^{+}$cells were detected that were not immediately adjacent to the basement membrane, suggesting downregulation of Ep-CAM expression upon commitment to spermatogenic differentiation. In neonatal ovaries, immature oocytes expressed moderate amounts of Ep-CAM (Fig. 4a). However, by adulthood, expression of the molecule was not detectable in the ovary. Several phases of follicle development, with Ep-CAM below detectable amounts at all stages are shown (Fig. 4f).

In addition to germ cells, ES cells were examined for the expression of Ep-CAM by flow cytometry and were found to be Ep-CAM ${ }^{+}$(Fig. 5a). When grown on untransfected $\left(\mathrm{Ep}-\mathrm{CAM}^{-}\right)$fibroblast feeder layers, ES cells segregate into distinct colonies by preferentially adhering to each other.
Since Ep-CAM is reported to be a homophilic adhesion molecule (Litvinov et al., 1994), it was investigated whether ectopic expression of Ep-CAM on feeder cells would disrupt ES cell morphology by increasing the adhesion between ES cells and the fibroblast monolayer. However, ES cell colony morphology was not affected by growth on Ep-CAM ${ }^{+}$ fibroblasts (Fig. 5b).

\section{Discussion}

Immunohistochemical experiments presented here document, for the first time, the expression of the homophilic adhesion molecule Ep-CAM in the embryonic and postnatal germ line of both sexes in mice. In the embryo, Ep-CAM is expressed by migratory PGCs and post-migratory germ cells. Anti-Ep-CAM antibody was found to label a fine network of processes on migratory PGCs to a degree previously not seen with other antibodies. At birth, Ep-CAM is expressed by germ cells in both the testis and ovary. At sexual maturity, expression of Ep-CAM is confined to spermatogonial stem cells adjacent to the basement membrane of the seminiferous tubules in the testis, whereas no Ep-CAM was detected in the ovary. Undifferentiated ES cells also express large amounts of immunoreactive EpCAM.

PGCs interact with each during migration by means of long, filopodial processes (Gomperts et al., 1994). The molecular basis of this interaction is not understood, but is presumably mediated by preferential PGC-PGC adhesion. Since PGC processes are Ep-CAM+ ${ }^{+}$, this molecule may play a role in this networking phenomena. Although it is well known that PGCs extend processes in vivo and in vitro, immunofluorescent staining with anti-Ep-CAM antibody revealed that these processes are much more extensive and complex than previously recognized. Although the function(s) of the filopodial networks made by PGCs are not yet know, it is possible that they play a role in migration, homing or aggregation.

After germ cells finish migration, they aggregate into tight clusters, an event hypothesized to be mediated by cell-cell adhesion molecule(s) on PGCs and somatic cells. In the present study, germ cell expression of Ep-CAM appeared to decrease from embryonic day 10.5 to day 12.5 , at the same time that germ cells aggregate. This finding may seem paradoxical, given that Ep-CAM is a cell-cell adhesion molecule (Litvinov et al., 1994). However, Ep-CAM expression has been shown to interfere with cadherin-based adhesion (Litvinov et al., 1997). Thus, the downregulation of Ep-CAM expression may facilitate a cadherin-based compaction of germ cell clusters. In addition, embryonic and adult germ cells have intercellular channels that may facilitate cell-cell adhesion (De Felici et al., 1989; Simon et al., 1997; Pepling and Spradling, 1998).

At embryonic day 12.5, the mesonephric tubules of both sexes expressed large amounts of Ep-CAM; this was expected since Ep-CAM is expressed by most epithelia (Litvinov et al., 1994). The mesonephros is adjacent to the early gonad. Despite the fact that the mesonephros frequently contains a large number of ectopic PGCs and that 

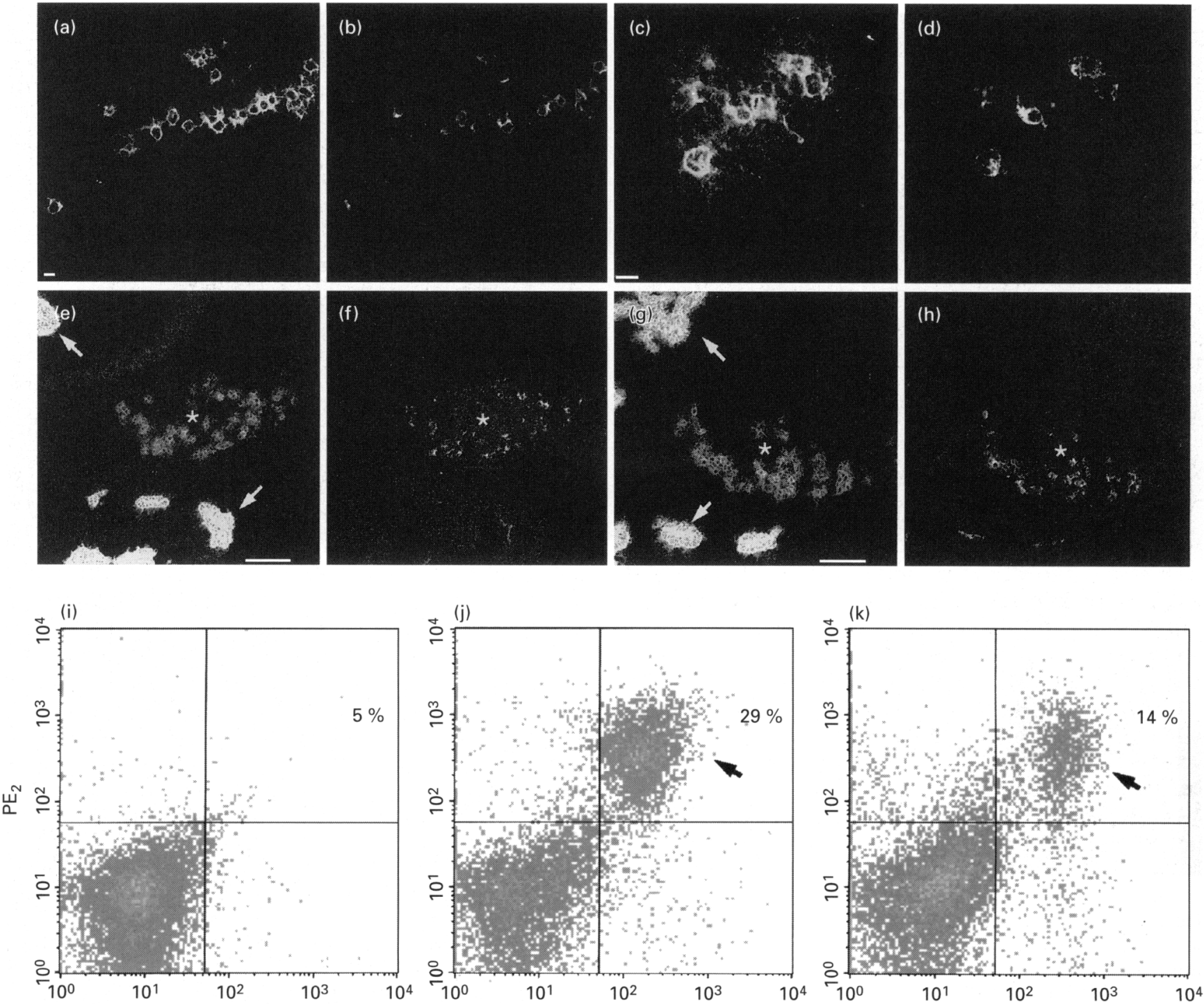

Fig. 2. Expression of the homophilic adhesion molecule, Ep-CAM, by embryonic day 10.5 and day 12.5 mouse primordial germ cells. (a-d) Sections of embryonic day 10.5 urogenital ridge double-stained for Ep-CAM (a,c: mAb C8.8) and germ cells (b,d: mAb TG-1). Sections of embryonic day 12.5 female $(e, f)$ and male $(g, h)$ gronads stained for Ep-CAM (e,g: mAb (.8.8) and germ cells (f,h: $m A b$ TG-1). Asterisks indicate the location of the gonad; arrows indicate positive staining of mesonephric and metanephric tubules. (i-k) Flow cytometric analysis staining for germ cells (anti-Fo, $y$-axis) and Ep-CAM (G8.8, $x$-axis) of embryonic day 12.5 forelimb (i), female gonad (j) and male gonad (k). FITC, fluorescein isothiocyanate. Arrow denotes primordial germ cells. Scale bars represent $10 \mu \mathrm{m}(\mathrm{a}-\mathrm{d})$ and 100$) \mu \mathrm{m}(\mathrm{c}-\mathrm{h})$

the mesonephric tubules are Ep-CAM+, germ cells are rarely found in association with the tubular epithelium. Therefore, Ep-CAM expression does not appear to be sufficient to attract or anchor PCCs. The role of Ep-CAM in the networking and aggregation of the early germ line will require genetic analysis.

The restriction of Ep-CAM expression to spermatogonia is reminiscent of E-cadherin expression in the testes of 8-dayold mice (Wu et al., 1993). The significance of this expression pattern is unclear, but it indicates that spermatogonial differentiation is accompanied by the immediate loss of EpCAM expression. Ep-CAM ${ }^{+}$E cadherin-transfected murine L (LEC) cells segregate to the outside of mixed cell clusters in vitro (Litvinov et al., 1997). Therefore, it is possible that Ep-
CAM plays an analogous role in compartmentalizing the cells of the seminiferous tubules, with Ep-CAM' spermatogonia segregating to the outside of the tubules. Alternatively, EpCAM expression may help to sustain the undifferentiated state of germ line stem cells by an adhesive or signalling mechanism. Regardless of the biological function of Ep-CAM in the adult testis, it should be considered a potentially useful immunocytochemical marker.

Embryonic stem cells are totipotent cells derived from cells of the inner cell mass. Embryonic day 11.5 PGCs are capable of forming ES-like cells (EG cells) in culture (Resnick et al., 1992). ES cells share many of the properties of the early germ line, including expression of alkaline phosphatase, the tyrosine kinase, Kit, and the transcription factor, Oct-4 (for 


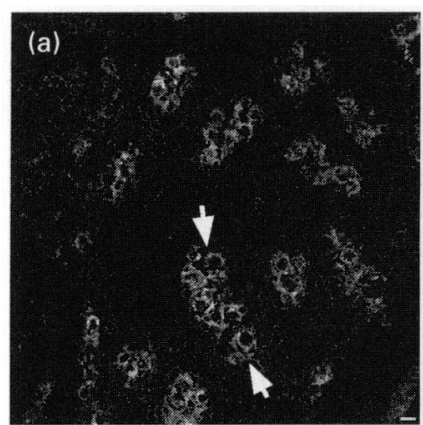

(b)
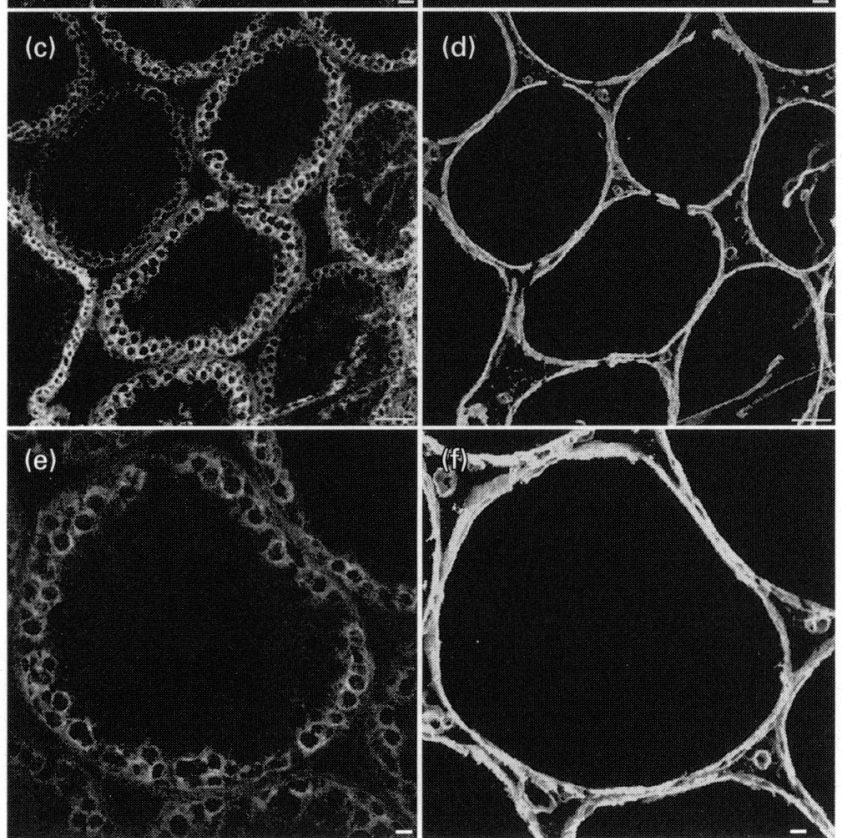

Fig. 3. Fxpression of the homophilic adthesion molecule, Fp-CAM, in neomatal and adult mouse testis. Neonatal testis stained for I:p-CAM (mAb C 8.8 ; a) or with omission of primary antibody (b). Arrows indicate staining of testis cord. Adult testis double stained for Fp-CAM $(C, 8.8 ; c,(e)$ and laminin as a basement membrane marker (anti-laminin; $d, f)$. Scale bars represent $10 \mu m(a, b, c, f)$ and $3(1) \mu m(c, d)$

review, see Yoem it al., 1996; Gardner and Brook, 1997). Therefore, the observation that ES cells express EP-CAM is not surprising, given the similarity of ES cells to the early germ line. In the present study, no differences in ts cell morphology were observed between plates of $\mathrm{Ep}-\mathrm{CAM}$ and wild-type (Ep-CAM) fibroblasts. This is probably because ES cells express additional adhesion molecules that favour self-adhesion (Tian et al., 1997). It is possible that expression of Ep-CAM by the transfected fibroblast cell line (clone D9) used was not sufficient to induce changes in ES cell morphology. Similarly, differences in I'CC behaviour have not been observed between plates of Ep-CAM and EpCAM fibroblasts (R. Anderson and C. Wylie, unpublished).

In conclusion, immunoreactive Ep-CAM is expressed in the mouse germ line throughout embryonic and fetal stages, indicating that it plays a morphogenetic or signalling role in the development of the gametes and totipotent cells. In addition, Ep-CAM is detectable on spermatogonia in the
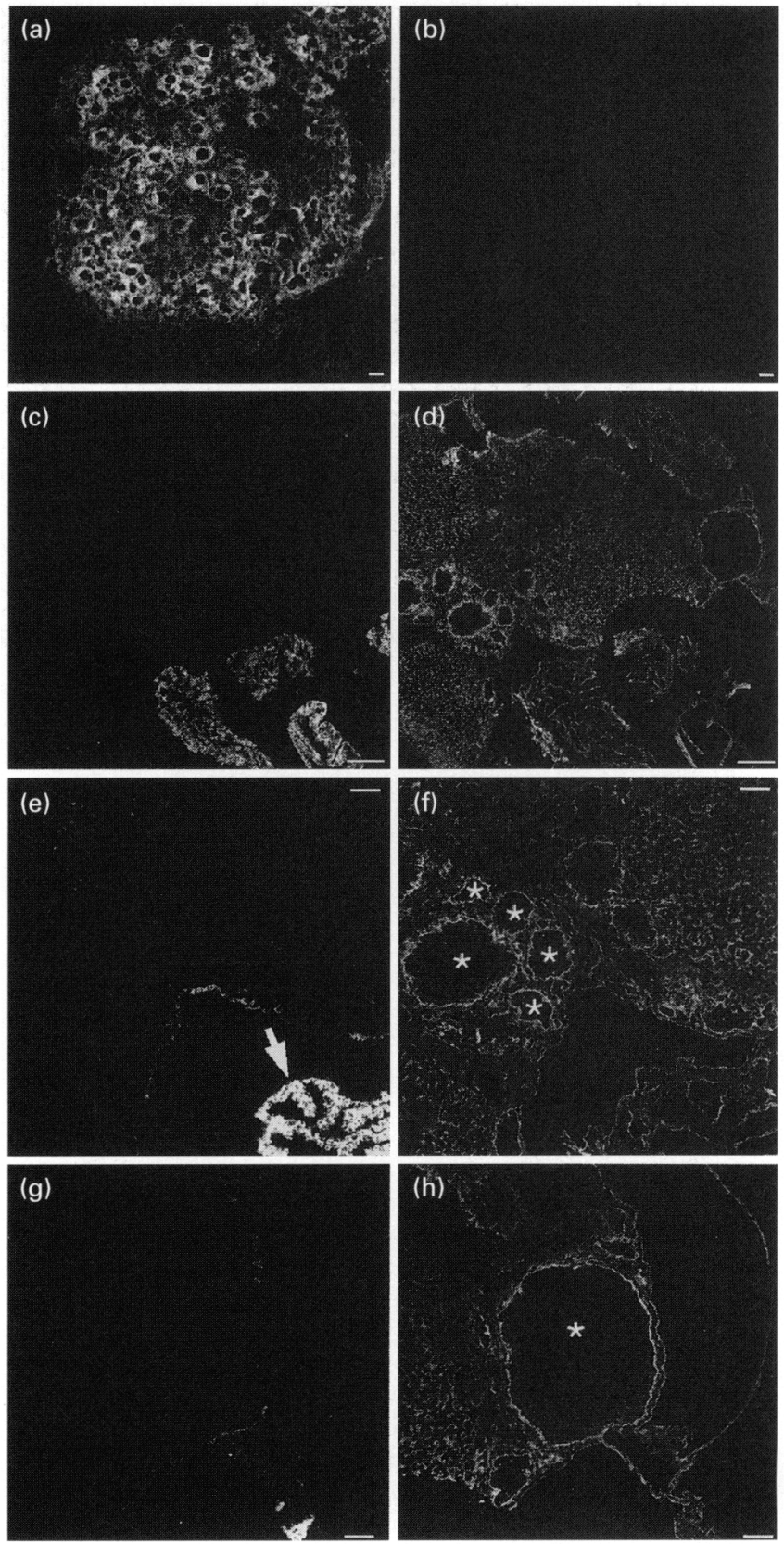

Fig. 4. Fipression of the homophilic adhesion molecule. Tp-C:M.11, in neonatal and adult mouse wary: Veomatal onary stained for lpCAM (mAb (is.s; a) or with omission of primary antibedy (b). Adult ovary double staned for lp-CAM $(C 88,8 ; 6,8)$ and laminin as a basement membrane marker (anti-laminin; $(1, i, 1)$. Arrow indicates positive staining of oviduct epithelium. Asterisk indicates lecation of developing oocytes (f) and ciratian follick (h) Siale bars reprenent 10) $\mathrm{rm}(\mathrm{a}, \mathrm{b}), 200 \mathrm{\mu m}(\mathrm{c}, \mathrm{d})$ and 3()$, \mathrm{m} \mathrm{m}(\mathrm{e}-\mathrm{h}\}$.

adult testis. Finally, Ep-CAM is expressed on the surface of ES cells. Given the widespread conservation of the Fp-CAM gene in vertebrates (Linnenbach of al., 1993), it is an attractive candidate for genetic analysis by targeted deletion in mice. Experiments of this kind should provide insight inte the function of Ep-CAM in the germ line and totipotent cells. 

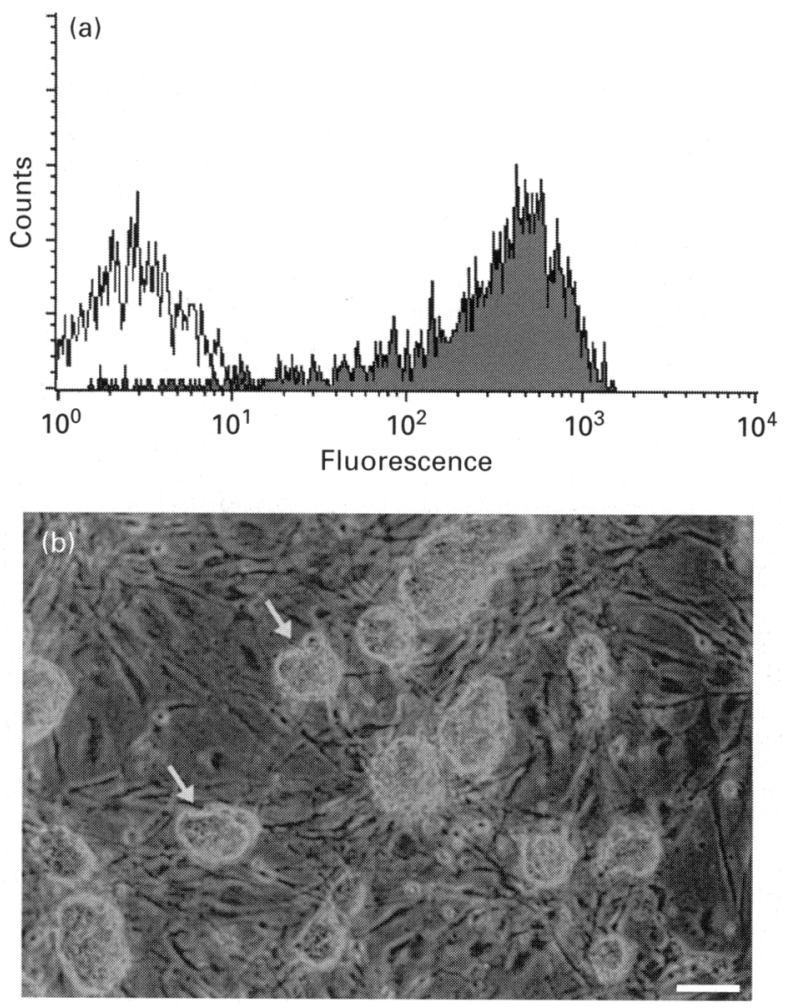

Fig. 5. Expression of the homophilic adhesion molecule, Ep-CAM, by mouse embryonic stem (ES) cells. (a) Flow cytometric analysis of ES cells stained for Ep-CAM (G8.8, dark curve) or with secondary antibody alone (grey curve). (b) ES cell colonies (arrows) have normal morphology when plated on Ep-CAM+ fibroblasts. Scale bar represents $30 \mu \mathrm{m}$.

The authors would like to thank the National Life and Health Insurance Medical Research Fund, the National Institute of Health (HD33440-01), the Harrison Fund and the Institute of Human Genetics for financial support of this work. The monoclonal antibody G8.8, developed by A. Farr, was obtained from the Developmental Studies Hybridoma Bank developed under the asupices of the NICHD, and maintained by the Department of Biological Sciences, University of Iowa, Iowa City, IA 52242. The authors would also like to thank P. Beverley for the gift of the TG-1 hybridoma, and Electra Coucouvanis for the gift of STO cells.

\section{References}

Anderson R, Garcia-Castro M, Heasman J and Wylie C (1998) Early stages in male germ cell differentiation in the mouse APMIS 106 127-133

Bergsagel P, Victor-Kobrin C, Timblin J, Trepel J and Kuehl W (1992) A murine cDNA encodes a pan-epithelial glycoprotein that is also expressed by plasma cells Journal of Imnunology 148 590-596

Cirulli V, Crisa L, Beattie G, Mally M, Lopez A, Fannon A, Ptasznik A, Inverardi L, Ricordi C, Deerinck T, Ellismann M, Reisfeld $R$ and Hayek $A$ (1998) KSA antigen Ep-CAM mediates cell-cell adhesion of pancreatic epithelial cells: morphoregulatory roles in pancreatic islet development journal of Cell Biology 140 1519-1534

Cooke J, Godin I, French-Constant C, Heasman J and Wylie C (1993) Culture and manipulation of germ cells Methods in Enzymology 225 37-77
Clayton L, McConnell M and Johnson M (1995) Control of surface expression of uvomorulin after activation of mouse oocytes Zygote 3 177-189

Cremer H, Lange R, Christoph A, Plomann M, Vopper G, Roes J, Brown R, Baldwin S, Kraemer P, Scheff S, Barthels D, Rajewsky $K$ and Wille $W$ (1994) Inactivation of the N-CAM gene in mice results in size reduction of the olfactory bulb and deficits in spatial learning Nature 267 455-459

De Felici M, Dolci S and Siracusa G (1989) Fetal germ cells establish coupling with follicle cells in vitro. Cell Differentiation and Development 28 65-70

Donovan P, Stott D, Cairns L, Heasman J and Wylie C (1986) Migratory and post-migratory mouse primordial germ cells behave differently in culture Cell 44 831-838

Gardner R and F Brook (1997) Reflections on the biology of embryonic stem (ES) cells International Journal of Developmental Biology 41 235-243

Gomperts M, Garcia-Castro M, Wylie C and Heasman J (1994) Interactions between primordial germ cells play a role in their migration in mouse embryogenesis Developntent 120 135-141

Hogan B, Beddington R, Constantini F and Lacy E (1994) Determining sex using molecular probes. In Manipulating the Mouse Embryo pp 382-383 Cold Spring Harbor Press, Cold Spring Harbor, NY

Hynes R (1996) Targeted mutations in cell adhesion genes: what have we learned from them? Developmental Biology 180 403-412

Lin L and DePhilip RM (1996) Sex-dependent expression of placental (P)cadherin during mouse gonadogenesis Anatomical Record 246 535-544

Linnenbach A, Seng B, Wu S, Robbins S, Scollon M, Pyrc J, Druck T and Huebner K (1993) Retroposition in a family of carcinoma-associated antigen genes Molecular Cell Biology 13 1507-1515

Litvinov S, Velders M, Bakker H, Flueren G and Warnaar S (1994) Ep-CAM: a human epithelial antigen is a homophilic cell-cell adhesion molecule Journal of Cell Biology 125 437-446

Litvinov S, Balzar M, Winter M, Bakker H, Briaire-de Bruijn I, Prins F, Fleuren G and Warnaar S (1997) Epithelial cell adhesion molecule (EpCAM) modulates cell-cell interactions mediated by classic cadherins fournal of Cell Biology 139 1337-1348

Moller C, Byskov A, Roth J, Celis J and Bock E (1991) NCAM in the developing mouse gonads and ducts Anatomy Embryology 184 541-548

Nelson A, Dunn R, Peach R, Aruffo A and Farr A (1996) The murine homolog of human Ep-CAM, a homotypic adhesion molecule, is expressed by thymocytes and thymic epithelial cells European journal of Inumunology 26 401-408

Newton S, Blaschuk O and Millette C (1993) N-Cadherin mediates Sertoli cell-spermatogenic cell adhesion Developmental Dynamics 197 1-13

Nocka K, Majumder S, Chabot B, Ray P, Cervone M, Bernstein A and Besmer P (1989) Expression of c-kit gene products in known cellular targets of $W$ mutations in normal and $W$ mutant mice - evidence for an impaired ckit kinase in mutant mice Genes and Development 3 816-826

Orth J and Jester $\mathbf{W}$ (1995) NCAM mediates adhesion between gonocytes and Sertoli cells in cocultures from testis of neonatal rats journal of Andrology 16 389-399

Pepling M and Spradling A (1998) Female mouse germ cells form synchronously dividing cysts Development 125 3323-3328

Resnick J, Bixler L, Cheng L and P Donovan (1992) Long-term proliferation of mouse primordial germ cells in culture Nature 359 550-551

Simon A, Goodenough D, Li E and Paul D (1997) Female infertility in mice lacking connexin 37 Nature 6 525-529

Stinnakre MG, Evans MJ, Willison KR and Stern PL (1981) Expression of Forssman antigen in the post-implantation mouse embryo Journal of Embryology and Experimental Morphology 61 117-131

Strand J, Hamilton L, Beavers G, Gambos L, Apelgren L, Taber J, Sportsman T, Bumol J, Sharp J and Gadski R (1989) Molecular cloning and characterization of a human adenocarcinoma/epithelial cell surface antigen complementary DNA Cancer Research 49 314-317

Tian L, Cat JW, O'Neill C and King NJ (1997) Expression of immunoglobulin superfamily cell adhesion molecules on murine embryonic stem cells Biology of Reproduction 57 561-568

Wu J-C, Gregory C and DePhilip R (1993) Expression of E-cadherin in immature rat and mouse testis and in rat Sertoli cell cultures Biology of Reproduction 49 1353-1361

Wylie C (1999) Germ cells Cell 96 165-174

Yeom Y, Fuhrmann G, Ovitt C, Brehm A, Kazuyuki O, Gross M, Hübner K and Schöler HR (1996) Germ line regulatory element of Oct-4 specific for the totipotent cycle of embryonic cells Development $122881-894$ 